\title{
Blockchain-Based Model to Improve the Performance of the Next-Generation Digital Supply Chain
}

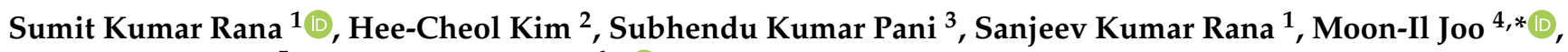 \\ Arun Kumar Rana ${ }^{5}$ and Satyabrata Aich ${ }^{6, *(D)}$ \\ 1 Computer Science and Engineering Department, Maharishi Markandeshwar (Deemed to be University), \\ Ambala 133207, India; sumitrana.cse@gmail.com (S.K.R.); dr.sanjeevrana@mmumullana.org (S.K.R.) \\ 2 College of AI Convergence/Institute of Digital Anti-Aging Healthcare/u-AHRC, Inje University, \\ Gimhae 50834, Korea; heeki@inje.ac.kr \\ 3 Department of Computer Applications, Krupajal Computer Academy, Bhubaneswar 751002, India; \\ pani.subhendu@gmail.com \\ 4 Institute of Digital Anti-Aging Healthcare, Inje University, Gimhae 50834, Korea \\ 5 Department of Electronics and Communication Engineering, Panipat Institute of Engineering and Technology, \\ Samalkha, Panipat 132102, India; ranaarun1@gmail.com \\ 6 Wellmatix Corporation Limited, Changwon 51395, Korea \\ * Correspondence: joomi@inje.ac.kr (M.-I.J.); satyabrataaich@gmail.com (S.A.); Tel.: +82-55-320-4027 (S.A.)
}

check for updates

Citation: Rana, S.K.; Kim, H.-C.; Pani, S.K.; Rana, S.K.; Joo, M.-I.; Rana, A.K.; Aich, S. Blockchain-Based Model to Improve the Performance of the Next-Generation Digital Supply Chain. Sustainability 2021, 13, 10008. https://doi.org/10.3390/su131810008

Academic Editor: Mark A. Bonn

Received: 17 July 2021

Accepted: 31 August 2021

Published: 7 September 2021

Publisher's Note: MDPI stays neutral with regard to jurisdictional claims in published maps and institutional affiliations.

Copyright: (c) 2021 by the authors. Licensee MDPI, Basel, Switzerland. This article is an open access article distributed under the terms and conditions of the Creative Commons Attribution (CC BY) license (https:// creativecommons.org/licenses/by/ $4.0 /)$.

\begin{abstract}
In the era of the fourth industrial revolution, all aspects of the industrial domain are being affected by emerging technologies. Digitalization of every process is taking place or under process. One of the most important components common to every domain is the supply chain process. Organizations employ a digital supply chain to track the delivery of their products or materials. The digital supply chain is still suffering from a few issues such as no provenance, less transparency, and a trust issue. Blockchain technology, one of the emerging technologies, can be integrated with the supply chain to deal with the existing issues and to improve its performance. In this paper, a model is proposed to integrate blockchain technology with the supply chain to improve performance. The proposed model uses the combination of the Ethereum blockchain and the interplanetary file system to maintain the traceability, transparency, and trustworthiness of the supply chain.
\end{abstract}

Keywords: blockchain; supply chain; digitalization; Ethereum blockchain; IPFS

\section{Introduction}

A supply chain is a series of steps in the production, transportation, storage, or distribution of a product [1]. Each stage may be handled by a single company, a group of companies, or a group of stakeholders. The global economy relies heavily on supply chains [2]. According to the International Trade Administration, supply chain transactions account for more than 76 percent of global trade [3]. To reduce production costs, large corporations outsource assembly lines to low-cost regions. The stages of the supply chain have been further divided, and an increasing number of affiliates are now in charge of them. Throughout the stages, supply chains have become more global, complex, and interdependent [4]. The survival of any organization depends on how good the synchronization is between different businesses functions involved in the process. One of the most important components of the business process is the supply chain. The supply chain is an integrated chain of different business functions, namely acquiring raw materials, delivery of products or services, etc. that are required to run a business. It also connects with all the stakeholders involved in the business. Effective supply chain management is a source of competitive advantage across various industries, including food, healthcare, IT, etc. [5]. If we look at recent history, several incidents related to the safety of agriproducts have occurred. A few examples are "poisonous ginger" that happened in China, infection in Hami melon due to 
listeria in the United States, and the epidemic of E. coli in Germany. Because of these incidents, countries are more attentive towards the traceability of agriproducts and regularly bring laws and regulations to the supply chain process. A traceability procedure must be attached with every supply chain so that in case of any outbreak, recall can be executed in an efficient manner and information can be passed to respective consumers. A traditional supply chain ecosystem is shown in Figure 1. In this type of approach, all the data related to all the processes is stored on a central database. This central database is taken care of by an administrator. Some limitations of this approach are mentioned in the following. As this uses a centralized server, if the central database server goes down, then the complete system will be down. This server is maintained by an administrator, if he is dishonest, he can manipulate the data without the knowledge of the stakeholders. These changes cannot be traced. So, this centralized approach also lacks traceability and transparency.

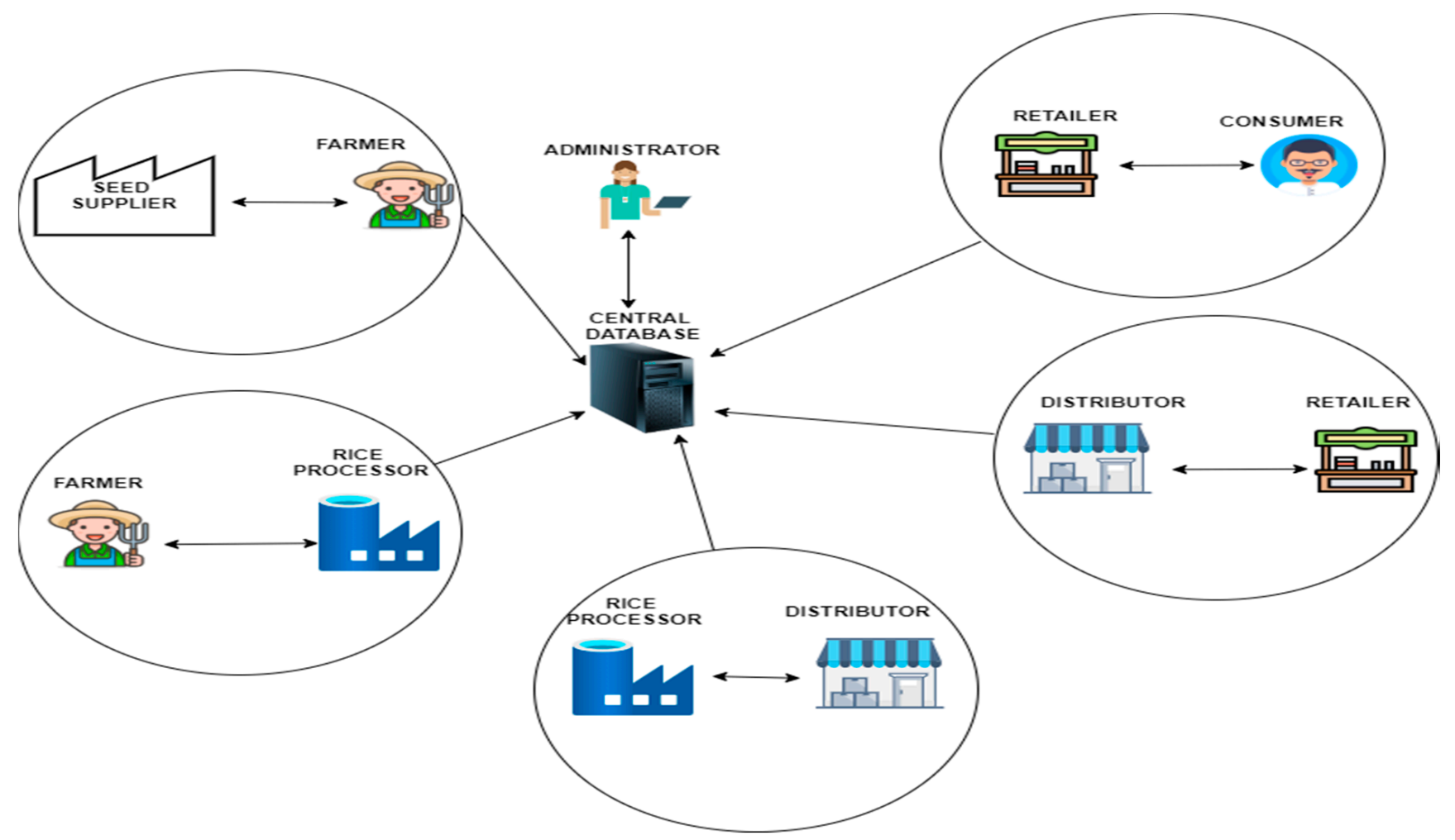

Figure 1. Traditional Supply Chain Ecosystem.

Major issues with the traditional supply chain ecosystem are traceability of the products, transparency for the stakeholders, trust in the collaborative system, and security from unauthorized modification of data. As there are multiple intermediaries in the traditional approach, trust and performance issues are common. There are multiple entities in the supply chain ecosystem such as farmers, distributors, retailers, etc. These can be in multiples and in different regions which makes it a complex network of entities. So, if there is an outbreak of any food-related contamination then traceability of the products is very difficult and time-consuming. Functional impact, social impact, economic impact, and the issues arising during the integration of emerging technologies with the supply chain ecosystem need to be investigated [6]. Second, the traditional supply chain ecosystem is completely centralized. This centralization brings trust issues in the collaborative environment where multiple entities are involved. In this centralized approach, data can be easily manipulated without the knowledge of other stakeholders. In the case of the food supply chain which delivers daily need products, traceability is of main concern because any carelessness may put the life or health of people at stake. Trust of the consumer should be the utmost priority for companies because trust issues in the supply chain process can cause significant losses. 
This process must be transparent to all the stakeholders of the process. So, to regain the trust of the consumer there must be some way to verify the origin of the food and to stop fraud [7].

These issues can be resolved if user data can be made accessible to all whereas alteration of data is not possible by anyone. To overcome these issues an emerging technology can be employed with the supply chain. Blockchain is the technology that can be used to remove the issues and improve the performance of the supply chain. It uses distributed ledger technology that has some features which can help in dealing with issues of the supply chain process. It is a distributed and immutable ledger that provides a trustable record that cannot be manipulated or tampered with by any entity. Transparency in the food supply chain and use of emerging technologies can improve the conventional supply chain ecosystem [8]. Data security and reliability, information interconnection and intercommunication, real-time sharing of hazardous-material information, and dynamic and credible whole-process tracing are missing in traditional systems [9]. Researchers are regularly working to provide a better understanding of blockchain innovation by presenting some key features and guidelines for accelerating blockchain adoption in industry [10].

This paper proposes a model supported by blockchain and smart contracts to trace the flow of data in the supply chain process of rice from its origin to the consumer. This model eliminates the need for intermediaries for different information sharing among the various stakeholders and advances the level of transparency, trust, and security. Different entities upload environmental data, rice growth data, buy and sell order data into the Interplanetary File System at the same time. The IPFS hash of the file is saved in smart contracts, which helps to solve the blockchain storage problem.

\section{Motivation and Contribution}

The motivation behind this research is the emergence of blockchain technology and its application in the supply chain ecosystem. Blockchain can provide better security, improved trust level, and avoid unauthorized modification due to the immutability feature. The main contributions of this paper are:

- Discussion of the recent publications on merging blockchain technology with the supply chain ecosystem.

- Investigation of the issues of the traditional supply chain and how these issues can be resolved by amalgamating blockchain technology.

- Presentation of a model that uses blockchain technology to improve the performance of the supply chain ecosystem.

- Proposition of a model that ensures transparency, traceability, and trustworthiness.

The remaining parts of the paper are structured as follows: In Section 2, research work of various authors related to blockchain application in the supply chain domain is explored. In Section 3, blockchain technology and its advantages in the supply chain domain are discussed. Section 4 presents a blockchain-supported model for the supply chain ecosystem. Section 5 concludes the paper and discusses future research directions.

\section{Related Study}

A. Shahid et al. presented a complete solution for the blockchain-based Agriculture and Food (Agri-Food) supply chain in our proposed solution. It makes use of the Ethereum blockchain network's key features of blockchain and smart contracts. Although blockchain ensures the immutability of data and records in the network, it still falls short of solving some major issues in supply chain management, such as the credibility of the parties involved, trading process accountability, and product traceability. Therefore, there is a need for a reliable system that ensures traceability, trust, and delivery mechanism in the Agri-Food supply chain. In the proposed system, all transactions are written to blockchain which ultimately uploads the data to the Interplanetary File Storage System (IPFS). The 
storage system returns a hash of the data which is stored on the blockchain and ensures an efficient, secure, and reliable solution [11].

A. Musamih et al. presented an Ethereum blockchain-based approach for efficient product traceability in the healthcare supply chain that uses smart contracts and decentralized off-chain storage. The smart contract ensures data provenance, eliminates the need for intermediaries, and provides all stakeholders with a secure, immutable transaction history. We present the system architecture as well as the detailed algorithms that govern our proposed solution's working principles. We test and validate the system, as well as present a cost and security analysis, to assess its effectiveness in improving traceability within pharmaceutical supply chains [12].

A. Kamilaris et al. looked at the impact of blockchain technology on agriculture and the food supply chain. They presented current projects and initiatives and discussed overall implications, challenges, and potential, all the while keeping a critical eye on the projects' maturity. Their findings showed that blockchain is a promising technology for a transparent food supply chain, with numerous ongoing initiatives in a variety of food products and food-related issues, but there are still many barriers and challenges that prevent it from gaining broader adoption among farmers and systems. Technical aspects, education, policies, and regulatory frameworks are all part of these challenges [13].

In business practice, there are numerous applications of BCT in supply chain management (SCM), and there is growing interest in this topic among academics. G. Blossey et al. combined these two perspectives on BCT in SCM to summarize the current state of the art and to identify areas for future research. A comprehensive framework of BCT using case clusters in SCM has been developed for this purpose, based on the unique features of BCT. The framework was used to examine 53 BCT applications in SCM that were discovered through a systematic literature review and a secondary dataset of blockchain-driven SCM innovations [14].

The potential application of blockchain technology and smart contracts to supply chain management was critically examined by S. Saberi et al. Government, community, and consumer pressures on local and global governments, communities, and consumers to meet sustainability goals prompted us to investigate how blockchain can address and aid supply chain sustainability. Part of this critical examination was to see how blockchains, a potentially disruptive technology still in its early stages of development, can overcome a variety of obstacles. Inter-organizational, intra-organizational, technical, and external barriers to blockchain technology adoption were discussed [15].

P. Gonczol et al. reviewed academic research and implementations of distributed ledgers on supply chains. They summarized the benefits and challenges of distributed supply chain organization and management, as well as the current state of research on the topic. They discussed the technical characteristics and maturity of various industrial projects, focusing on industrial practices and use cases. Their goal was to evaluate the utility of blockchains in the supply chain domain and to lay the groundwork for practitioners and researchers to focus their future projects on improving the technology and its applications [16].

A blockchain-IoT-based food traceability system (BIFTS) was proposed by Y.P.Tsang et al. The authors integrated the novel deployment of blockchain, IoT technology, and fuzzy logic into a total traceability shelf-life management system for perishable food management. Lightweight and vaporized characteristics were deployed in the blockchain to address the needs for food traceability, while an integrated consensus mechanism that considered shipment transit time, stakeholder assessment, and shipment volume was developed. The blockchain data flow was then synchronized with the deployment of IoT technologies at the level of traceable resource units [17].

K.Salah et al. proposed a method for tracking and tracing soybeans across the agricultural supply chain that used the Ethereum blockchain and smart contracts to efficiently perform business transactions. Their proposed solution eliminates the need for a trusted centralized authority, intermediaries, and transaction records, resulting in increased effi- 
ciency and safety while maintaining high integrity, reliability, and security. The proposed solution focused on the use of smart contracts to govern and control all interactions and transactions between all stakeholders in the supply chain ecosystem. All transactions were recorded and stored in the blockchain's immutable ledger with links to a decentralized file system (IPFS), thus providing to all a high level of transparency and traceability into the supply chain ecosystem in a secure, trusted, reliable, and efficient manner [18].

Current academic and industrial frontiers on blockchain application in the supply chain, logistics, and transportation management were presented by M. Pournader et al. The authors conducted a systematic review of the literature and discover four major clusters in the co-citation analysis: technology, trust, trade, and traceability/transparency. They used an inductive method of reasoning to discuss the emerging themes and applications of blockchains for supply chains, logistics, and transportation for each cluster, based on the pool of articles included in it. Finally, they went over the main topics for future blockchain research and its applications in industry and services [19].

H. Treiblmaier et al. presented a framework based on four established economic theories, namely principal-agent theory (PAT), transaction cost analysis (TCA), the resourcebased view (RBV), and network theory. The authors discussed closing the current research gap on the potential implications of the blockchain for supply chain management (SCM) (NT). These theories can be used to generate research questions that were both theory-based and industry-relevant [20].

Khanfar et al. conducted a systematic review to find the applications of blockchain technology in the manufacturing industries and its associated supply chain. They touched on various parameters for evaluation of social and economic performance. They discussed the benefits of employing blockchain technology in the sustainable process of manufacturing. They also proposed a model that illustrates how blockchain technology can contribute to sustainable manufacturing [21].

M.P. Kramer et al. studied the distinctions between traditional and blockchain supported food supply chain networks in the domain of agri-food. They identified the major coordination mechanisms that are supported differently depending on the selected blockchain platform types, based on an extensive literature exploration. The findings were then compared to agri-food industry use cases. The authors claimed that the properties of supply chain coordination mechanisms vary based on the blockchain platform chosen, and that the chosen platform has an impact on the economic performance of the proposed business model [22].

J. Nurgazina et al. aimed to highlight the existing practical uses of distributed ledger technology and internet of things in food supply chain. The authors also mentioned the problems of implementation and relevant research topics for future research, to contribute to the United Nations Sustainable Development Goals. The content of over sixty scholarly articles was reviewed in a comprehensive literature analysis, outlining the strategies to solve the difficulties of scalability, security, and privacy of distributed ledger technology. The main issues in its implementation were increased cost, absence of regulatory framework, and the energy consumption of distributed ledger technology [23].

V. Varriale et al. investigated the effects of employing emerging technologies in improving the sustainable process. Three parameters of supply chain process were compared in different virtual environments. In the first case, the conventional process was used without emerging technologies. In the second case, the same process was carried out with blockchain and internet of things. The findings revealed that performance was better when emerging technologies were employed [24].

A. Park et al. checked the long-term reliability of supply chain management (SCM) in terms of environmental and social factors. They evaluated up to which percentage blockchain technology can better reliability parameters for supply chains. They compiled the papers by analyzing with respect to different components of the literature. They discovered that research on the influence of distributed ledger technology on reliability has been expanding regularly [25]. 
The pandemic situation around the world exposed the hidden vulnerabilities of the supply chain ecosystem. The primary concern of researchers around the world was to increase the capability of the supply chain process. W. Yin et al. went through an extensive study of the literature to find all the possibilities that could improve the capability of the supply chain process. They analyzed the use of blockchain technology in the supply chain and found that blockchain technology had a great potential that could impact the capability of supply chain in multidimensional domains [26].

V. Varriale et al. explored the use case of distributed ledger technology for a reliable supply chain ecosystem. They synthesized the current state of development and proposed a methodology for the future development. Implications of the research were also investigated. They also explained the advantages of integrating blockchain technology for the development of a reliable supply chain [27].

After the study and analysis of the supply chain ecosystem related literature survey, we found that the domain of the supply chain supported by emerging technologies has been less explored as compared to other domains. We also found that research papers are mostly theoretical and very little information is provided at the implementation level. Some of the authors did try to explain the implementation point of view. Therefore, this study was designed to explore the use of blockchain technology in the supply chain ecosystem. We also investigated the implementation perspective. We used the Ethereum blockchain with proof of authority consensus algorithm, the smart contract written in solidity, and the interplanetary file system for decentralized storage. As the digital environment and technology are evolving, the supply chain ecosystem should be updated with the new emerging technologies.

\section{Blockchain Technology for the Supply Chain}

Blockchain technology is one of the emerging technologies that will affect most of the domains which are working in a collaborative environment. It is a distributed ledger technology in which different entities can communicate with each other in a peer-to-peer network without any middle entity [28]. It provides multiple features (shown in Figure 2) that can bring trust and transparency in a collaborative environment.

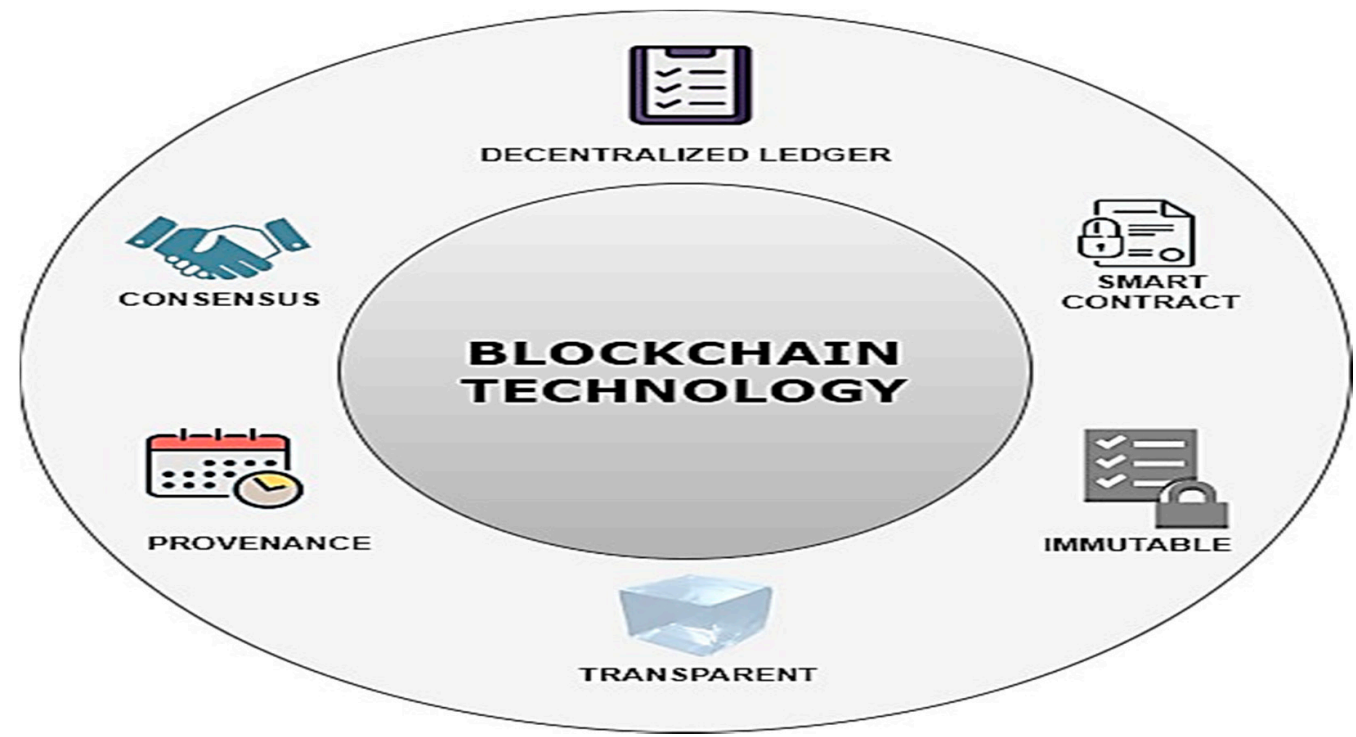

Figure 2. Blockchain Technology features.

Because of its decentralized nature, no single entity can control it. Consensus is the agreement of all the stakeholders on the execution of a transaction. It provides a provenance feature by which the history of any transaction can be traced back. All the transactions are updated in the distributed ledger so that these are visible to all the connected nodes of the 
network [29]. One of the most important features that make it tamper-proof is immutability. Once any transaction is written in the distributed ledger then no one can edit or delete this transaction. It also supports smart contracts. A smart contract is a computer program that contains the logic of a contract between two or more entities. This contract is executed when certain conditions are met [30].

\section{Advantages of Integrating Blockchain Technology in the Supply Chain}

- Transparent procurement: Whenever any company goes for procurement, it looks for some middle entity that can do procurement for them. In the traditional approach, it is very difficult to track the actual volume or quantity among all the stakeholders such as partner firms, subsidiaries, etc. The blockchain's distributed ledger can help in this process of procurement. This ledger is shared among all the stakeholders and continuously updated so that each transaction is visible to all. Companies can easily verify their orders by a distributed ledger. Companies have to recruit many people for auditing purposes only. With the help of blockchain, auditing can be done within minutes without the involvement of so many people [31].

- Smart contracts for payments: Normally whenever a task is completed an invoice is generated and sent to the client for the payment of the task. As this process is operated manually it can take more time than the expectation of the concerned party. By using blockchain, smart contracts can be integrated into this process. Whenever a task is completed, its invoice is generated automatically and sent to the client digitally. After the verification of the invoice, the smart contract will be triggered, and payment is automatically credited into the account of the company. This is how the integration of blockchain can improve the process of payment settlement [32].

- No more fraud by rogues: With blockchain, a decentralized ledger is shared among all the connected nodes. All the transactions are updated in this ledger which is reflected in the ledgers of every connected node. If a rogue tries to execute some transaction for his benefit, he will not succeed. Because blockchain employs a consensus feature, no transaction can be executed without the agreement of all the stakeholders [33].

- Provenance tracking: If there is any case where historical data of any transaction is needed to resolve any discrepancy then blockchain technology can be useful. The blockchain-supported solution has a feature of provenance tracking. It means any transaction can be traced back to its time of origin from its current instance [34].

- Immutable transactions: There can be multiple transactions executed by authorized nodes. If any attacker node wants to delete or modify the transactions, he would not be able to do this. Because blockchain has an immutability feature once a transaction is written in the decentralized ledger nobody can delete or modify the transaction. Even the administrator cannot delete or update the previously executed transactions. As hash function is used in blockchain technology, even a small change in the data can change the hash of the data as shown below. If only $\mathrm{H}$ is replaced by $\mathrm{h}$, then the hash is completely changed [35].

Hash $($ Hello $)=185 f 8 d b 32271 f e 25 f 561 a 6 f c 938 b 2 e 264306 e c 304 e d a 518007 d 1764826381969$

Hash (hello) $=2 c f 24 d b a 5 f b 0 a 30 e 26 e 83 b 2 a c 5 b 9 e 29 e 1 b 161 e 5 c 1 f a 7425 e 73043362938 b 9824$

\section{Methodology and Proposed Model}

We have proposed a model that uses blockchain technology and associated concepts for the betterment of the supply chain ecosystem. In the traditional system, the supply chain ecosystem begins with the producer of the product and ends with the consumer of the product. A producer produces a product and sells it to a processor for processing of data at the next level of the supply chain ecosystem. Then that processor processes the raw product and converts it to a usable form. Then the processor sells it to a distributor at the next level of the supply chain system. Then the distributor sells it to retailers at the next level. Finally, the retailer sells the product to the consumer of the product. In this 
traditional approach, a centralized approach is used. Only communicating parties at each level share the data and stakeholders at other levels are not able to see the transactions. In this traditional approach, the history of the product is not traceable so the end user cannot verify the source of the product. Also, the data are not trustworthy because this approach is centralized. In our proposed model, blockchain technology is employed which is decentralized in nature. Decentralized means no single entity will be able to control the complete supply chain ecosystem. There will be no client server approach as it supports a peer-to-peer network model. Any entity can communicate with any other entity on the network without involving any intermediaries. All the data will be stored in a decentralized manner. If some of the systems are down, then it will not affect the performance of the network. All the transactions are visible to all stakeholders. Historical data is also retained in the blockchain so the consumer can easily verify the source of the product. In our proposed model, blockchain is utilized to provide security to documents such as purchase orders, invoices, etc. uploaded by the respective stakeholder of the supply chain by using Ethereum blockchain and IPFS.

The blockchain supported supply chain ecosystem is shown in Figure 3. The main components of the proposed model are digital documents, Ethereum blockchain, interplanetary file system, and system users. All the components are integrated so that these components can exchange information with each other. These components are discussed in the following.

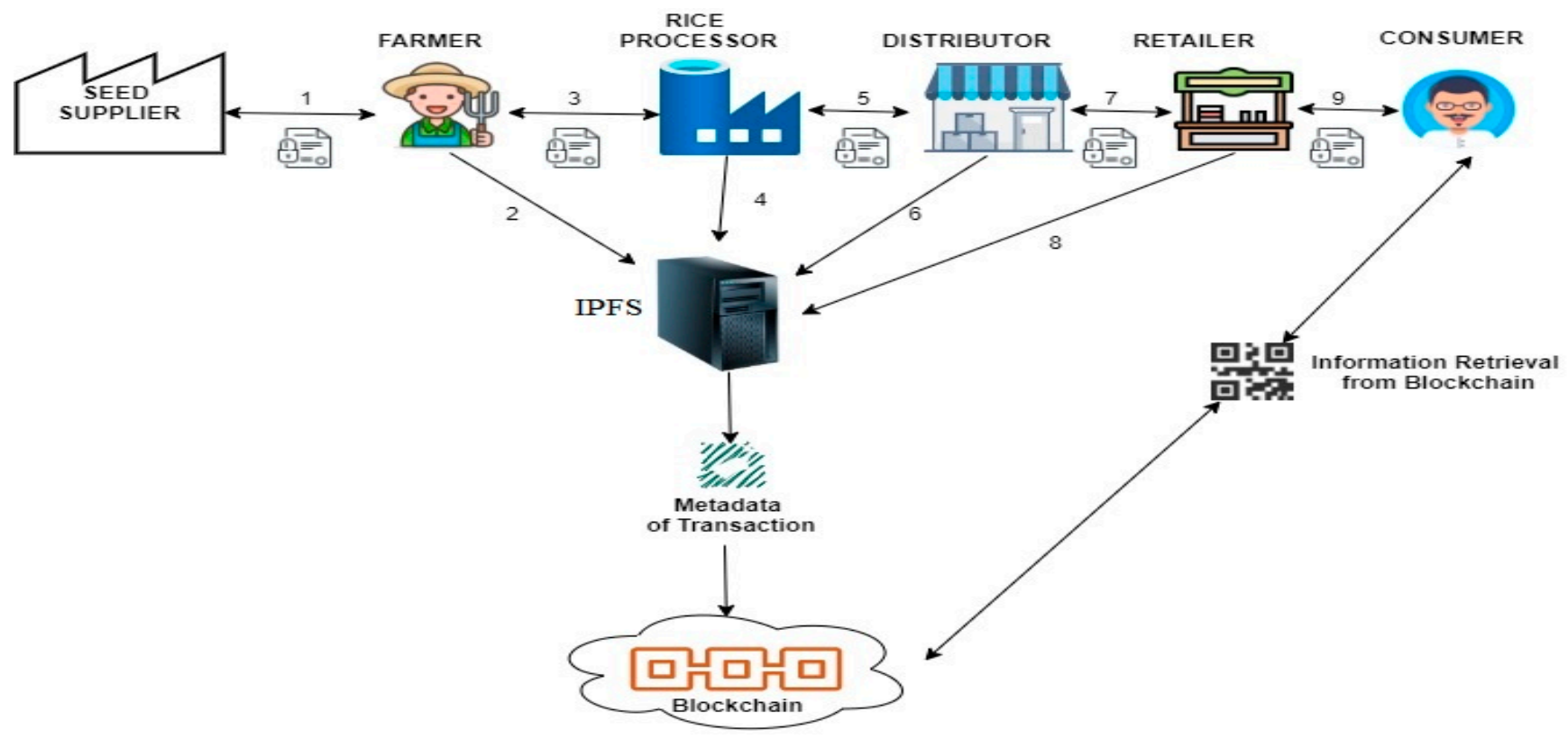

Figure 3. Blockchain Supported Supply Chain Ecosystem.

(1) Digital Documents: These are the documents like purchase orders, dispatch advice, payment advice, etc. that are uploaded by different stakeholders such as the manufacturer, distributor, retailer, etc. during the process of production of goods to their delivery to consumers [36]. This is one of the most important components of the proposed model. Multiple entities will access these documents during the process. so, the security and integrity of these documents are of prime concern because tampered with or manipulated documents can lead to fraud. Such fraud can cause a big loss to stakeholders that will hurt their trust. So, decentralized access control is provided with the help of the Ethereum blockchain which is the next component of the proposed model.

(2) Ethereum Blockchain: Ethereum is an open source blockchain that is used to develop decentralized application. It uses a ledger that stores the transactions. This technology can be used to store the access transactions of documents. Because of the immutability property of the blockchain, document access transactions cannot be modified. 
Documents cannot be stored on the Ethereum blockchain because the size of the block in the blockchain is very small. Off-the-chain storage is required to store the digital documents. IPFS, which is discussed next, is used to fulfil our need for off-the-chain storage.

(3) IPFS: Interplanetary File System is a distributed system for storing and accessing information such as documents, websites, applications, etc. It uses a content addressing scheme by which any file can be addressed by its content. Whenever a file is stored on IPFS, a unique cryptographic hash is returned. This hash is used to address or identify that file. This hash also avoids duplication of data because the hash remains the same for two files with the same data. Digital documents will be stored in encrypted form on IPFS in the proposed model [37].

(4) System User: The system user can be any stakeholder of the process such as the consumer, retailer, distributor, or manufacturer. Each user of the system is provided with a pair of keys that can be used while accessing the digital documents related to them.

\section{Work-Flow Analysis}

We now take the example of supplying rice from farmer to consumer. In this example, first, farmers buy seeds from the seed supplier. Then he grows seeds on his farm. After cutting the crops he will upload the cropped detail (CD) document which contains details like seed type, seed supplier and crops, etc. on the IPFS. Information related to this document will be stored on the blockchain in the form of a transaction. Then rice processor can place a buy order (BO) directly to the farmer after checking all the details of seeds and crops. After receiving the unprocessed rice, the processor processes the rice and uploads the document which contains the processing details. The distributor places a $\mathrm{BO}$ to the processor. After receiving the processed rice, the distributor checks for a BO from the retailer. Then the distributor ships the order to various retailers. The distributor uploads a document that contains shipment details of the order. After receiving the rice, the retailer sells the rice to the consumer. The consumer can check the complete data related to the rice from its beginning date to end date. Each entity can communicate with another entity with the help of a smart contract. This model will use the proof of authority consensus mechanism. According to this consensus, only selected nodes are given the responsibility of validating the transactions. For example, if any entity wants to upload data, then the request will be sent to the validator node to validate the transaction. The validator node will receive a notification regarding the transaction. If the validator finds it to be a legitimate transaction, then he approves the transaction. If the validator finds it to be non-authentic then he can decline the transaction. The entity-relationship diagram is shown in Figure 4.

The sequence diagram for the proposed model is shown in Figure 5. This diagram shows the sequence of function execution during the supply chain process of rice from its origin to its delivery to the consumer. 


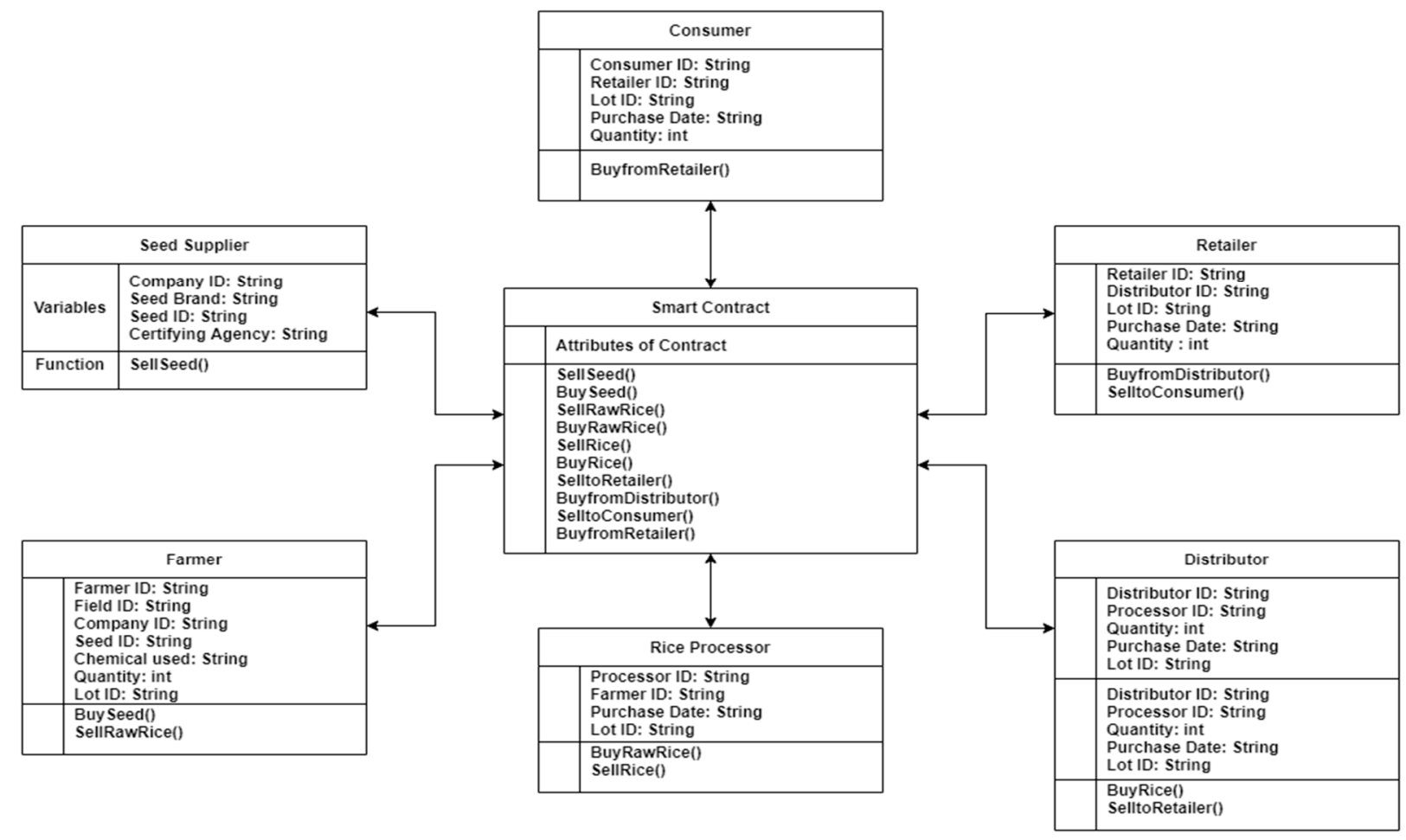

Figure 4. Entity Relationship Diagram for the proposed model.

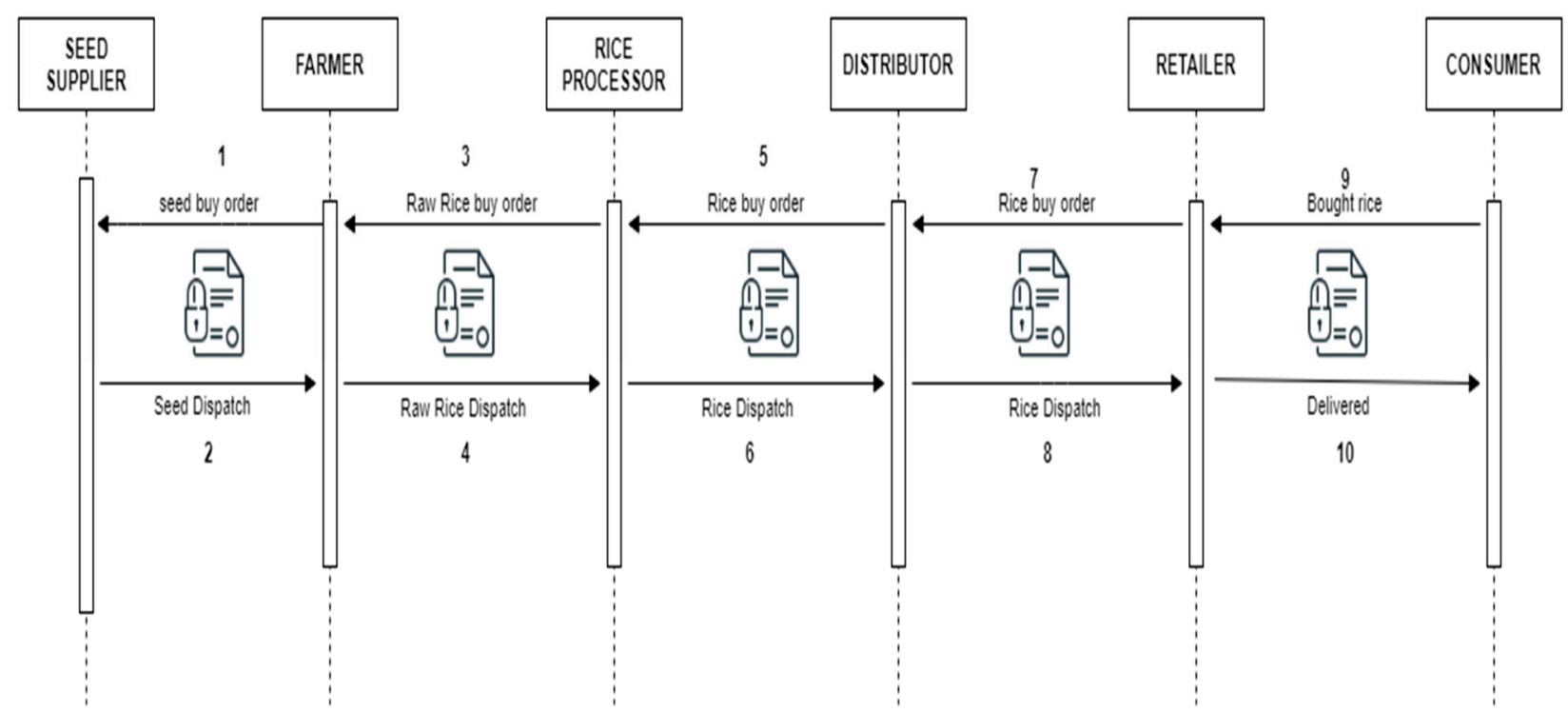

Figure 5. Sequence Diagram for the proposed model.

Two algorithms for communication between seed supplier, farmer, and rice processor are given below. Algorithm 1 illustrates the smart contract communication between the seed supplier and the farmer. Seed is requested from the seed supplier. If the seed sale is agreed and seed prices are paid then the seed request is granted and a notification is sent to the farmer. Otherwise, the request is rejected, and notification of the cancelled request is sent to the farmer. In Algorithm 2, smart contract communication between the farmer and the rice processor is shown. In this algorithm a similar process is followed. If the raw rice sale is agreed and the price is paid then request of the rice processor is granted or else rejected. 


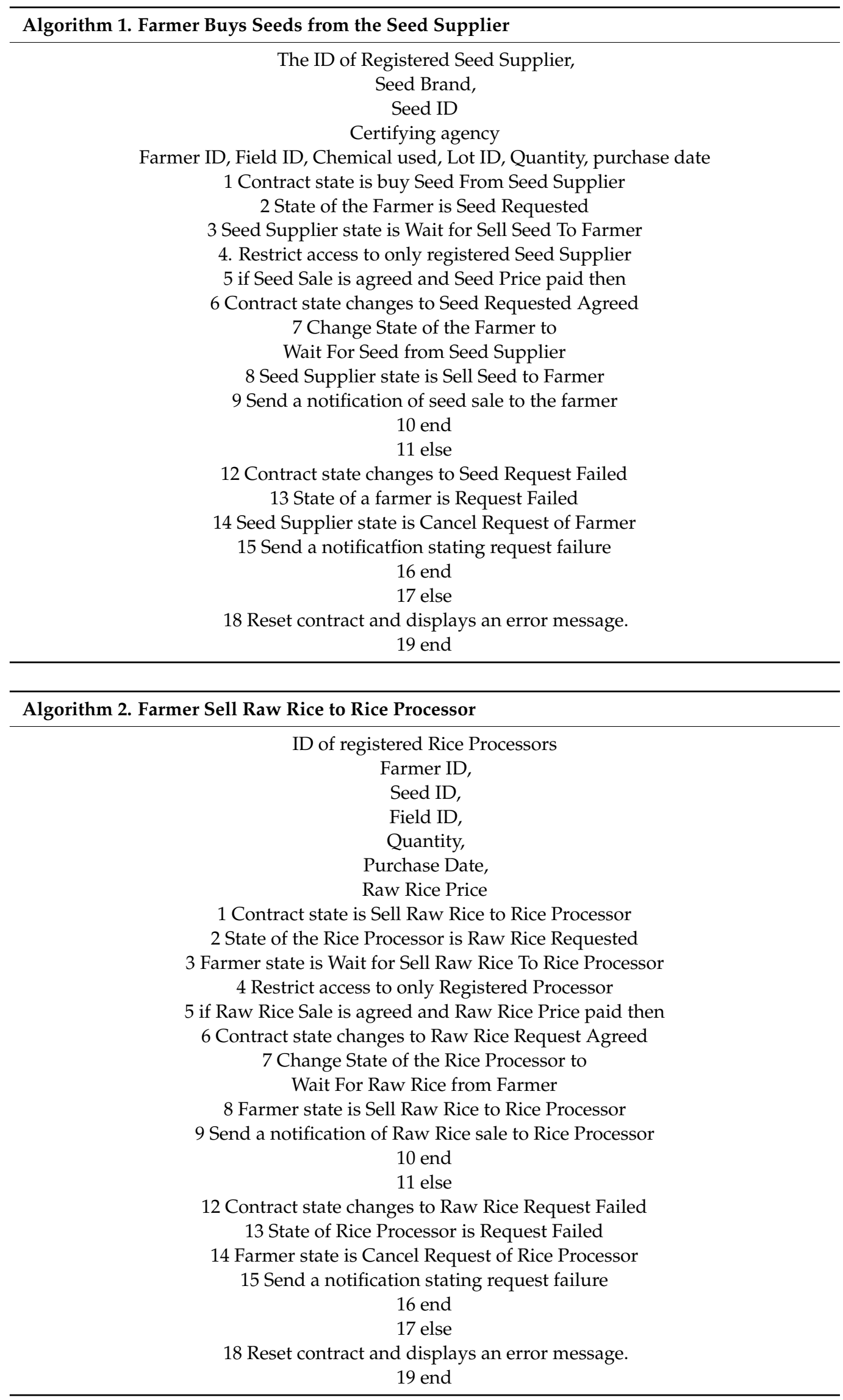


The plus of this approach over the standard food supply chain systems is shown in Table 1.

Table 1. Outcomes of the proposed model over traditional models.

\begin{tabular}{ccc}
\hline Attributes & Proposed System (with Blockchain) & $\begin{array}{c}\text { Traditional Method (without } \\
\text { Blockchain) }\end{array}$ \\
\hline Structure & Decentralized & Centralized \\
\hline Storage & Distributed & Centralized \\
\hline Provenance & History of transactions are available & $\begin{array}{c}\text { Historical information is not } \\
\text { recorded }\end{array}$ \\
\hline Trmutability & $\begin{array}{c}\text { Once written, even admin cannot } \\
\text { change the transaction information }\end{array}$ & $\begin{array}{c}\text { The administrator can easily } \\
\text { manipulate the information }\end{array}$ \\
\hline Consensus & $\begin{array}{c}\text { Trust in a collaborative environment } \\
\text { is increased }\end{array}$ & $\begin{array}{c}\text { Trust issues are there with a } \\
\text { centralized approach }\end{array}$ \\
\hline
\end{tabular}

The proposed model takes full advantage of the blockchain's benefits, overcomes the problem of depending on core companies to gather information, and makes the information interaction between all nodes more transparent. The issue of unauthorized tampering is avoided because the blockchain information cannot be tampered with. Furthermore, utilizing the blockchain's consensus process, the "trust problem" between different links in the conventional food supply chain is overcome.

In previous work, some authors did not give the name of the consensus algorithm and some authors used the byzantine fault tolerant algorithm such as proof of work. The proof of work algorithm is time consuming as it takes approximately 10-60 min to add a transaction to a blockchain. It needs to solve a mathematical puzzle to make a transaction so resource consumption is high. It has a risk of $51 \%$ of attack. With $51 \%$ attack, if an entity is able to control $51 \%$ or more nodes of the blockchain network then the proof of work consensus algorithm will be of no use. In our proposed model, the proof of authority consensus algorithm is used. In this algorithm, a few selected nodes can act as validator to validate the transactions. Validators are nodes that have the same identity on the network as they do in the public notary database. They reveal their true identities willingly. Validators demonstrate their commitment to the network over time. They should be willing to invest their money and risk their reputation to maintain the network running smoothly. In our model, representatives from the seed supplier or rice processor company or a distributor can act as validator nodes. All the transactions will be validated by these validator nodes. The time required to generate a block is predictable and less than the proof of work algorithm because rather than involving all the nodes in the validation of transactions, only preselected validator nodes will verify the transaction. This model encourages collaboration and verification among grain supply chain stakeholders, integrates resources efficiently, and optimizes benefits. After a thorough study of the literature, a comparative analysis of traditional models with the proposed model is shown in Figure 6. As per our observation, we found that the proposed system is far better than the traditional method in all attributes. These scores represent the presence or absence of features such as trust, immutability, provenance, and consensus. A high score represents a significant presence of the particular feature in the proposed model and a low score for the traditional models represents the absence or nominal presence of the particular feature. No experts were used for the evaluation. Comparative analysis compares traditional models and the proposed model and determines the presence and absence of the above-mentioned features. Comparison is done on the basis of various attributes such as trust, immutability, provenance, and consensus. The proposed model is more trustworthy as it works in a decentralized manner because of blockchain technology. There is no central authority that 
can control the entire network but the model uses immutable distributed ledger therefore once the transaction is written in the ledger it cannot be deleted or modified. This model has a provenance feature. It means transactions can be traced back from their current state to their origin state and also has a consensus mechanism whereby every transaction will be executed by the agreement of the authorized nodes. High scores represent a significant presence of the respective parameter and low scores represent the absence of the same.

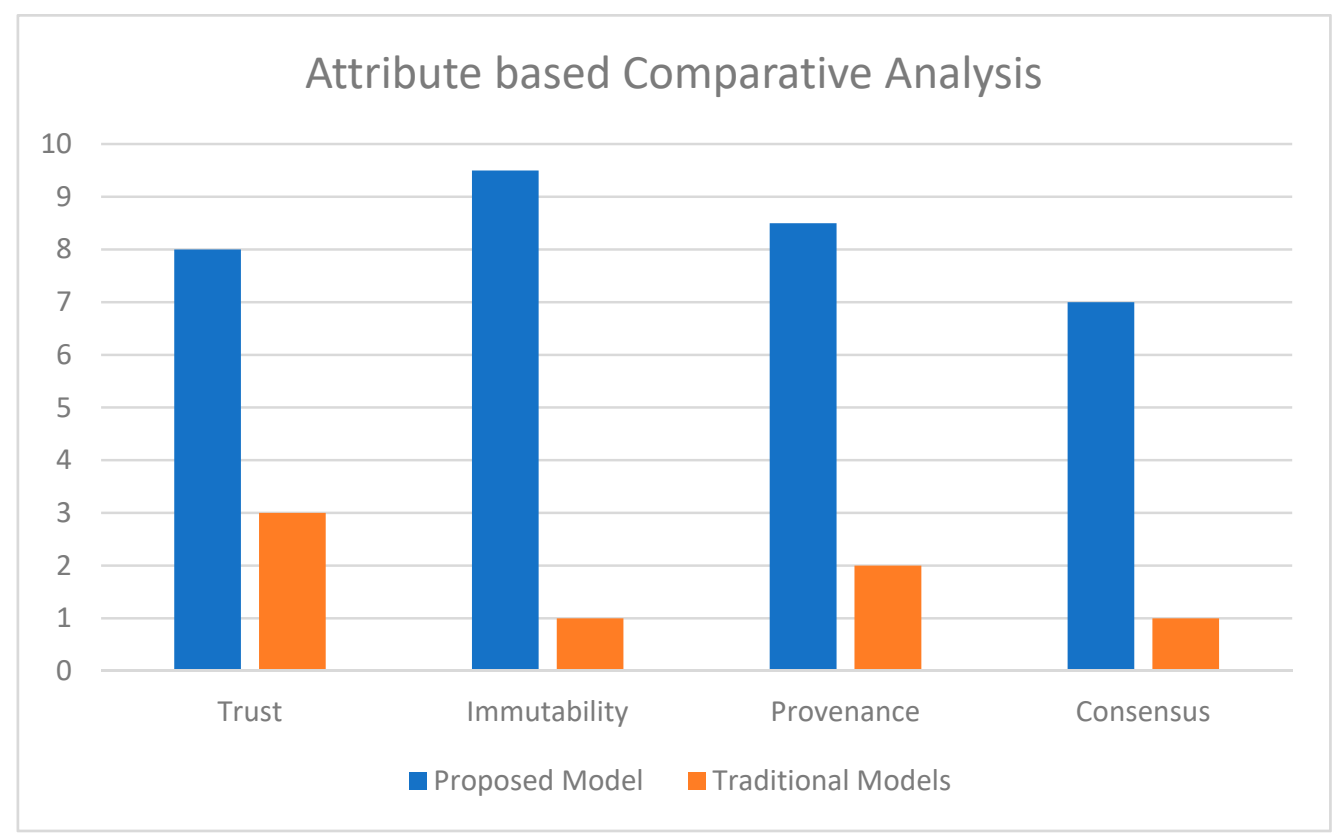

Figure 6. Attribute based comparative analysis.

The limitations of the blockchain technology-based model are that it has constraints such as the number of transactions per second, latency in transactions, and the amount of data in transactions. Regular updates in blockchain technology frameworks will surely improve the performance of these types of models in the future.

\section{Conclusions}

In a multi-stakeholder supply chain, maintaining product and process integrity is a significant challenge. Data fragmentation, a lack of reliable provenance, and a variety of protocol regulations across multiple distributions and processes plague many current solutions. Blockchain, among other technologies, has emerged as a leading technology because it provides secure traceability and control, immutability, and stakeholder trust in a low-cost IT solution [38]. The main goal of this article was to answer two research questions. First, what are the most important issues of the traditional supply chain ecosystem and second, how can these issues be resolved by integrating blockchain technology with the supply chain ecosystem. To determine the issues, we explored multiple research articles of this domain. To answer the second question, the various benefits of integration blockchain technology with the supply chain were explored and a model proposed. The proposed model employs the Ethereum blockchain with the proof of authority consensus algorithm and an interplanetary file system. This proposed model improves the performance by removing intermediaries and making payment processing via smart contract. We presented different aspects of the proposed model such as entity-relationship diagrams, sequence diagrams, and algorithms for smart contract communication. We presented how this integration of blockchain technology in the supply chain can bring trust, transparency, and security by immutability. This model can be applied to different supply chain models of various domains with slight amendments. 
Author Contributions: Conceptualization, S.K.R. (Sumit Kumar Rana), A.K.R., S.K.P., S.K.R. (Sanjeev Kumar Rana); methodology, S.K.R. (Sumit Kumar Rana), A.K.R., S.K.P., S.K.R. (Sanjeev Kumar Rana); validation, S.K.R. (Sumit Kumar Rana), A.K.R., S.K.P., S.K.R. (Sanjeev Kumar Rana); formal analysis, S.K.R. (Sumit Kumar Rana), A.K.R., S.K.P., S.K.R. (Sanjeev Kumar Rana); data curation, S.K.R. (Sumit Kumar Rana), A.K.R., S.K.P., S.K.R. (Sanjeev Kumar Rana); writing-original draft preparation, S.K.R. (Sumit Kumar Rana), A.K.R., S.K.P., S.K.R. (Sanjeev Kumar Rana); writing-review and editing, S.K.R. (Sumit Kumar Rana), A.K.R., and S.A.; supervision, S.A. and H.-C.K.; project administration, S.A., M.-I.J., and H.-C.K.; funding acquisition, S.A., M.-I.J., and H.-C.K. All authors have read and agreed to the published version of the manuscript.

Funding: This research was supported by This research was supported by the Basic Science Research Program through the National Research Foundation of Korea (NRF) funded by the Ministry of Education (NRF2021R1I1A1A01050306) and by the Ministry of Science, ICT, and Future Planning (NRF2017R1D1A3B04032905) and This work was supported by the Commercializations Promotion Agency for R\&D Outcomes (COMPA) grant funded by the Korean Government (Ministry of Science and ICT)" (R\&D project No.1711139492).

Conflicts of Interest: The authors declare no conflict of interest.

\section{References}

1. Omar, I.A.; Jayaraman, R.; Debe, M.S.; Omar, M. Automating Procurement Contracts in the Healthcare Supply Chain Using Blockchain Smart Contracts. IEEE Access 2021, 9, 37397-37409. [CrossRef]

2. Kumar, A.; Sharma, S.; Goyal, N.; Singh, A.; Cheng, X.; Singh, P. Secure and energy-efficient smart building architecture with emerging technology IoT. Comput. Commun. 2021, 176, 207-217. [CrossRef]

3. Chang, S.E.; Chen, Y. When Blockchain Meets Supply Chain: A Systematic Literature Review on Current Development and Potential Applications. IEEE Access 2020, 8, 62478-62494. [CrossRef]

4. Wang, L.; Xu, L.; Zheng, Z.; Liu, S.; Li, X.; Cao, L.; Li, J.; Sun, C. Smart Contract-Based Agricultural Food Supply Chain Traceability. IEEE Access 2021, 9, 9296-9307. [CrossRef]

5. Rana, A.K.; Sharma, S. Enhanced energy-efficient heterogeneous routing protocols in WSNs for IoT application. Int. J. Eng. Adv. Technol. 2019, 9, 4418-44250.

6. Lezoche, M.; Hernandez, J.E.; Diaz, M.; Panetto, H.; Kacprzyk, J. Agri-food 4.0: A survey of the supply chains and technologies for the future agriculture. Comput. Ind. 2020, 117, 103187. [CrossRef]

7. Gopi, K.; Mazumder, D.; Sammut, J.; Saintilan, N. Determining the provenance and authenticity of seafood: A review of current methodologies. Trends Food Sci. Technol. 2019, 91, 294-304. [CrossRef]

8. Astill, J.; Dara, R.; Campbell, M.; Farber, J.; Fraser, E.; Sharif, S.; Yada, R. Transparency in food supply chains: A review of enabling technology solutions. Trends Food Sci. Technol. 2019, 91, 240-247. [CrossRef]

9. Zhao, Z.; Dong, Y. Blockchain-Based Safety Management System for the Grain Supply Chain. IEEE Access 2020, 8, 36398-36410.

10. Della Valle, F.; Oliver, M. Blockchain Enablers for Supply Chains: How to Boost Implementation in Industry. IEEE Access 2020, 8 , 209699-209716. [CrossRef]

11. Shahid, A.; Almogren, A.; Javaid, N. Blockchain-Based Agri-Food Supply Chain: A Complete Solution. IEEE Access 2020, 8 , 69230-69243. [CrossRef]

12. Musamih, A.; Salah, K.; Member, S.; Jayaraman, R. A Blockchain-Based Approach for Drug Traceability in Healthcare Supply Chain. IEEE Access 2021, 9, 9728-9743. [CrossRef]

13. Kamilaris, A.; Fonts, A.; Boldv, F.X.P. The rise of blockchain technology in agriculture and food supply chains. Trends Food Sci. Technol. 2019, 91, 640-652. [CrossRef]

14. Blossey, G.; Eisenhardt, J.; Hahn, G. Blockchain Technology in Supply Chain Management: An Application Perspective. In Proceedings of the 52nd Hawaii International Conference on System Sciences, Grand Wailea, HI, USA, 8-11 January 2019; Volume 6, pp. 6885-6893.

15. Saberi, S.; Kouhizadeh, M.; Sarkis, J.; Shen, L. Blockchain technology and its relationships to sustainable supply chain management. Int. J. Prod. Res. 2018, 57, 2117-2135. [CrossRef]

16. Gonczol, P.; Katsikouli, P.; Herskind, L.; Dragoni, N. Blockchain Implementations and Use Cases for Supply Chains-A Survey. IEEE Access 2020, 8, 11856-11871. [CrossRef]

17. Tsang, Y.P.; Choy, K.L.; Wu, C.H.; Ho, G.T.S.; Lam, H. Blockchain-Driven IoT for Food Traceability With an Integrated Consensus Mechanism. IEEE Access 2019, 7, 129000-129017. [CrossRef]

18. Salah, K.; Nizamuddin, N.; Jayaraman, R.; Omar, M. Blockchain-Based Soybean Traceability in Agricultural Supply Chain. IEEE Access 2019, 7, 73295-73305. [CrossRef]

19. Pournader, M.; Shi, Y.; Seuring, S.; Koh, S.C.L. Blockchain applications in supply chains, transport and logistics: A systematic review of the literature. Int. J. Prod. Res. 2020, 58, 2063-2081. [CrossRef]

20. Treiblmaier, H. The impact of the blockchain on the supply chain: A theory-based research framework and a call for action. Supply Chain Manag. Int. J. 2018, 23, 545-559. [CrossRef] 
21. Khanfar, A.A.A.; Iranmanesh, M.; Ghobakhloo, M.; Senali, M.; Fathi, M. Applications of Blockchain Technology in Sustainable Manufacturing and Supply Chain Management: A Systematic Review. Sustainability 2021, 13, 7870. [CrossRef]

22. Kramer, M.P.; Bitsch, L.; Hanf, J. Blockchain and Its Impacts on Agri-Food Supply Chain Network Management. Sustainability 2021, 13, 2168. [CrossRef]

23. Nurgazina, J.; Pakdeetrakulwong, U.; Moser, T.; Reiner, G. Distributed Ledger Technology Applications in Food Supply Chains: A Review of Challenges and Future Research Directions. Sustainability 2021, 13, 4206. [CrossRef]

24. Varriale, V.; Cammarano, A.; Michelino, F.; Caputo, M. Sustainable Supply Chains with Blockchain, IoT and RFID: A Simulation on Order Management. Sustainability 2021, 13, 6372. [CrossRef]

25. Park, A.; Li, H. The Effect of Blockchain Technology on Supply Chain Sustainability Performances. Sustainability 2021, $13,1726$. [CrossRef]

26. Yin, W.; Ran, W. Theoretical Exploration of Supply Chain Viability Utilizing Blockchain Technology. Sustainability 2021, $13,8231$. [CrossRef]

27. Varriale, V.; Cammarano, A.; Michelino, F.; Caputo, M. The unknown potential of blockchain for sustainable supply chains. Sustainability 2020, 12, 9400. [CrossRef]

28. Rana, A.K.; Sharma, S. Industry 4.0 manufacturing based on IoT, cloud computing, and big data: Manufacturing purpose scenario. In Advances in Communication and Computational Technology; Springer: Singapore, 2021; pp. 1109-1119.

29. Rana, A.K.; Sharma, S. Contiki Cooja Security Solution (CCSS) with IPv6 Routing Protocol for Low-Power and Lossy Networks (RPL) in Internet of Things Applications. In Mobile Radio Communications and 5G Networks; Springer: Singapore, 2021; pp. 251-259.

30. Buterin, V. A next generation smart contract \& decentralized application platform- Ethereum white paper. White Paper 2013, 3, $1-36$.

31. Khan, S.; Loukil, F.; Guegan, C.G.; Benkhelifa, E.; Bani hani, A. Blockchain smart contracts: Applications, challenges and future trends. In Peer-to-Peer Networking and Applications; Springer: Canada, 2021. Available online: https://www.ncbi.nlm.nih.gov/ pmc/articles/PMC8053233/ (accessed on 30 August 2021).

32. Norvill, R.; Pontiveros, B.B.; State, R.; Cullen, A. IPFS for reduction of chain size in Ethereum. In Proceedings of the IEEE Conference on IoT, Green Computing, Smart Data, Blockchain, Congress on Cybernetics, Halifax, NS, Canada, 30 July-3 August 2018; pp. 1121-1128.

33. Dias, D.; Benet, J. Distributed Web Applications with IPFS in ICWE; Springer LNCS: Basel, Switzerland, 2016 ; pp. 616-619.

34. Kouhizadeh, M.; Saberi, S.; Sarkis, J. Blockchain technology and the sustainable supply chain: Theoretically exploring adoption barriers. Int. J. Prod. Econ. 2020, 231, 107831. [CrossRef]

35. Nandi, S.; Sarkis, J.; Hervani, A.A.; Helms, M.M. Redesigning Supply Chains using Blockchain-Enabled Circular Economy and COVID-19 Experiences. Sustain. Prod. Consum. 2020, 27, 10-22. [CrossRef]

36. Kurpjuweit, S.; Schmidt, C.G.; Klöckner, M.; Wagner, S.M. Blockchain in Additive Manufacturing and its Impact on Supply Chains. J. Bus. Logist. 2019, 42, 46-70. [CrossRef]

37. Saurabh, S.; Dey, K. Blockchain technology adoption, architecture, and sustainable agri-food supply chains. J. Clean. Prod. 2020, 284, 124731. [CrossRef]

38. Swain, S.; Peter, O.; Adimuthu, R.; Muduli, K. Blockchain technology for limiting the impact of pandemic: Challenges and prospects. In Computational Modeling and Data Analysis in COVID-19 Research; CRC Press: Boca Raton, FL, USA, 2021; pp. 165-186. 